\title{
A NATURAL MAP FROM A QUANTIZED SPACE ONTO ITS SEMICLASSICAL LIMIT AND A MULTI-PARAMETER POISSON WEYL ALGEBRA
}

\author{
SEI-QWON OH
}

\begin{abstract}
A natural map from a quantized space onto its semiclassical limit is obtained. As an application, we see that an induced map by the natural map is a homeomorphism from the spectrum of the multi-parameter quantized Weyl algebra onto the Poisson spectrum of its semiclassical limit.
\end{abstract}

\section{INTRODUCTION}

A quantized coordinate ring $\mathcal{O}_{q}(V)$ of an affine variety $V$ is informally a deformation of its classical coordinate ring $\mathcal{O}(V)$. Let us replace the parameter $q \in \mathcal{O}_{q}(V)$ by an indeterminate $t$. If a central element $h$ of $\mathcal{O}_{t}(V)$ is non-zero, non-unit and non-zero-divisor and the factor algebra $\mathcal{O}_{t}(V) / h \mathcal{O}_{t}(V)$ is commutative then $\mathcal{O}_{t}(V) / h \mathcal{O}_{t}(V)$ is a Poisson algebra with Poisson bracket defined by $\{\bar{a}, \bar{b}\}=\overline{h^{-1}(a b-b a)}$. In such case, the Poisson algebra $\mathcal{O}_{t}(V) / h \mathcal{O}_{t}(V)$ is called a semiclassical limit of $\mathcal{O}_{t}(V)$ and $\mathcal{O}_{t}(V)$ is called a quantization of the Poisson algebra $\mathcal{O}_{t}(V) / h \mathcal{O}_{t}(V)$. Here we study a class $\mathcal{O}_{q}(V)$ of quantized algebras containing many cases such as [5], 7], 8], [11, [12, [13. Our aim of this paper is to construct a natural map from $\mathcal{O}_{q}(V)$ onto its semiclassical limit. As an application, we prove that this natural map induces a homeomorphism between the spectrum of quantized Weyl algebra and the Poisson spectrum of its semiclassical limit.

The multiparameter quantized Weyl algebra $R_{n}^{Q, \Lambda}$ was constructed by G. Maltsiniotis [9]. The spectrum Spec $R_{n}^{Q, \Lambda}$ was described by M. Akhavizadegan and David A. Jordan [1] and K. Goodearl and E. Letzter [7] proved that $R_{n}^{Q, \Lambda}$ satisfies the Dixmier-Moeglin equivalence. Here we find a semiclassical limit $A_{1}$ of $R_{n}^{Q, \Lambda}$ and prove that $A_{1}$ satisfies the Poisson Dixmier-Moeglin equivalence. Moreover we construct a natural homeomorphism from $\operatorname{Spec} R_{n}^{Q, \Lambda}$ onto P. Spec $A_{1}$ such that its restriction to the primitive spectrum $\operatorname{Prim} R_{n}^{Q, \Lambda}$ is also a homeomorphism onto the Poisson primitive spectrum P. Prim $A_{1}$ by applying the main result of this paper. This gives an affirmative answer for $R_{n}^{Q, \Lambda}$ for the conjecture posted in [5, 9.1]: Assume that $k$ is an algebraically closed field of characteristic zero, and let $A$ be a generic quantized coordinate ring of an affine algebraic variety $V$ over $k$. Then $A$ should be a member of a flat family of $k$-algebras with semiclassical limit $\mathcal{O}(V)$, such that the primitive spectrum of $A$ is homeomorphic to the space of symplectic cores in $V$, with respect to the semiclassical limit Poisson structure. Further,

Date: August 31, 2015.

2010 Mathematics Subject Classification. 17B63, $16 \mathrm{~S} 36$.

Key words and phrases. Poisson algebra, quantized algebra, semiclassical limit, quantized Weyl algebra.

The author is supported by National Research Foundation of Korea Grant 2012-007347. 
there should be compatible homeomorphisms from the primitive spectrum of A onto the Poisson primitive spectrum of $\mathcal{O}(V)$ and from the spectrum of $A$ onto the Poisson spectrum of $\mathcal{O}(V)$.

In the first section, we see a method how to construct a natural map from a quantized space into its semi-classical limit which can induce a homeomorphism and the ideas are illustrated by the quantized coordinate ring $\mathcal{O}_{q}\left(\mathbb{C}^{2}\right)$ of affine 2 -space. In the second section, we construct a semiclassical limit $A_{1}$ of $R_{n}^{Q, \Lambda}$ called a multiparameter Poisson Weyl algebra. Then we find its Poisson spectrum and Poisson primitive spectrum by modifying the proofs given by M. Akhavizadegan and David A. Jordan [1] and prove that $A_{1}$ satisfies the Poisson Dixmier-Moeglin equivalence.

Assume throughout the paper that the base field is the complex number field $\mathbb{C}$ and that all algebras considered have unity.

Definition 0.1. (1) A commutative algebra $A$ over $\mathbb{C}$ is said to be a Poisson algebra if there exists a bilinear product $\{-,-\}$ on $A$, called a Poisson bracket, such that $(A,\{-,-\})$ is a Lie algebra and $\{a b, c\}=a\{b, c\}+\{a, c\} b$ for all $a, b, c \in A$.

(2) An ideal $I$ of a Poisson algebra $A$ is said to be a Poisson ideal if $\{I, A\} \subseteq I$. A Poisson ideal $P$ is said to be Poisson prime if, for all Poisson ideals $I$ and $J, I J \subseteq P$ implies $I \subseteq P$ or $J \subseteq P$. If $A$ is noetherian then a Poisson prime ideal of $A$ is a prime ideal by [4, Lemma 1.1(d)].

For an ideal $I$ of a Poisson algebra $A$, the largest Poisson ideal $\mathcal{P}(I)$ contained in $I$ is called the Poisson core of $I$. If $I$ is prime then $\mathcal{P}(I)$ is prime by [3, 3.3.2]. A Poisson ideal $P$ of $A$ is said to be Poisson primitive if $P=\mathcal{P}(M)$ for some maximal ideal $M$ of $A$. Note that Poisson primitive is Poisson prime.

Definition 0.2. (1) Let $R$ be an algebra. The spectrum of $R$, denoted by Spec $R$, is the set of all prime ideals of $R$ equipped with the Zariski topology. The primitive spectrum, denoted by Prim $R$, is the subspace of Spec $R$ consisting of all primitive ideals of $R$.

(2) Let $A$ be a Poisson algebra. The Poisson spectrum of $A$, denoted by $\mathrm{P} . \operatorname{Spec} A$, is the set of all Poisson prime ideals of $A$ equipped with the Zariski topology. The Poisson primitive spectrum of $A$, denoted by P.Prim $A$, is the subspace of P. Spec $A$ consisting of all Poisson primitive ideals of $A$. If $A$ is noetherian then $\mathrm{P}$. Spec $A$ is a subspace of $\operatorname{Spec} A \operatorname{since}$ Poisson prime is prime.

\section{NATURAL MAP}

Notation 1.1. Let $t$ be an indeterminate.

(1) Assume that $\mathbf{K}$ is an infinite subset of the set $\mathbb{C} \backslash\{0,1\}$.

(2) Assume that $\mathbb{F}$ is a subring of the ring of regular functions on $\mathbf{K} \cup\{1\}$ containing $\mathbb{C}\left[t, t^{-1}\right]$. That is,

$$
\mathbb{C}\left[t, t^{-1}\right] \subseteq \mathbb{F} \subseteq\{f / g \in \mathbb{C}(t) \mid f, g \in \mathbb{C}[t] \text { such that } g(1) \neq 0, g(\lambda) \neq 0 \forall \lambda \in \mathbf{K}\} .
$$

(3) Let $\mathbb{F}\left\langle x_{1}, \ldots, x_{n}\right\rangle$ be the free $\mathbb{F}$-algebra on the set $\left\{x_{1}, \ldots, x_{n}\right\}$. A finite product $\mathbf{x}$ of $x_{i}$ 's (repetitions allowed) is called a monomial. For each $i=1, \ldots, r$, let $f_{i}$ be an $\mathbb{F}$-linear combination of monomials

Set

$$
f_{i}=\sum_{\mathbf{x}} a_{\mathbf{x}}^{i}(t) \mathbf{x}, \quad a_{\mathbf{x}}^{i}(t) \in \mathbb{F} .
$$

$$
A=\mathbb{F}\left\langle x_{1}, \ldots, x_{n}\right\rangle / I
$$


where $I$ is the ideal of $\mathbb{F}\left\langle x_{1}, \ldots, x_{n}\right\rangle$ generated by $f_{1}, \ldots, f_{r}$. That is, $A$ is the $\mathbb{F}$-algebra generated by $x_{1}, \ldots, x_{n}$ subject to the relations

$$
f_{1}, \ldots, f_{r}
$$

Note that $A$ is also a $\mathbb{C}$-algebra since $\mathbb{C} \subseteq \mathbb{F}$.

(4) Assume that $t-1$ is a nonzero, nonunit and non-zero-divisor of $A$ such that the factor $A_{1}=A /(t-1) A$ is commutative. Denote by $\gamma_{1}$ the canonical homomorphism of $\mathbb{C}$-algebras

$$
\gamma_{1}: A \longrightarrow A_{1} \text {. }
$$

Note, by [2, III.5.4], that the commutative $\mathbb{C}$-algebra $A_{1}$ is a Poisson algebra with Poisson bracket

$$
\left\{\gamma_{1}(a), \gamma_{1}(b)\right\}=\gamma_{1}\left((t-1)^{-1}(a b-b a)\right)
$$

for all $a, b \in A$. The Poisson algebra $A_{1}$ is said to be a semiclassical limit of $A$.

(5) For each $\lambda \in \mathbf{K}$, let $A_{\lambda}$ be the $\mathbb{C}$-algebra generated by $x_{1}, \ldots, x_{n}$ subject to the relations

$$
\left.f_{1}\right|_{t=\lambda}, \ldots,\left.f_{r}\right|_{t=\lambda}
$$

where $\left.f_{i}\right|_{t=\lambda}=\sum_{\mathbf{x}} a_{\mathbf{x}}^{i}(\lambda) \mathbf{x}$. Note that $a_{\mathbf{x}}^{i}(\lambda)$ is a well-defined element of $\mathbb{C}$ by (1.1) and that there exists a canonical homomorphism of $\mathbb{C}$-algebras

$$
\gamma_{\lambda}: A \longrightarrow A_{\lambda}
$$

such that $\gamma_{\lambda}(t)=\lambda, \gamma_{\lambda}\left(x_{i}\right)=x_{i}$ for $i=1, \ldots, n$.

(6) Set

$$
\widehat{A}=\prod_{\lambda \in \mathbf{K}} A_{\lambda}
$$

Let

$$
\pi_{\lambda}: \widehat{A} \longrightarrow A_{\lambda}
$$

be the canonical projection onto $A_{\lambda}$ for each $\lambda \in \mathbf{K}$ and let $\gamma$ be the homomorphism of $\mathbb{C}$-algebras

$$
\gamma: A \longrightarrow \widehat{A}, \gamma(a)=\left(\gamma_{\lambda}(a)\right)_{\lambda \in \mathbf{K}}
$$

Thus $\pi_{\lambda} \gamma=\gamma_{\lambda}$ for each $\lambda \in \mathbf{K}$.

Note that $\gamma(t-1)$ is an invertible element of $\widehat{A}$ since $1 \notin \mathbf{K}$.

(7) Assume that there exists an $\mathbb{F}$-basis $\left\{\xi_{i} \mid i \in I\right\}$ of $A$ such that

$$
\left\{\gamma_{1}\left(\xi_{i}\right) \mid i \in I\right\}, \quad\left\{\gamma_{\lambda}\left(\xi_{i}\right) \mid i \in I\right\}
$$

are $\mathbb{C}$-bases of $A_{1}$ and $A_{\lambda}$, respectively, for each $\lambda \in \mathbf{K}$.

Hence every element $a \in A$ is expressed uniquely by

$$
a=\sum_{i} a_{i}(t) \xi_{i}, \quad a_{i}(t) \in \mathbb{F}
$$

and, for each $\lambda \in \mathbf{K}$,

$$
\gamma_{\lambda}(a)=\sum_{i} a_{i}(\lambda) \gamma_{\lambda}\left(\xi_{i}\right), \quad \gamma_{1}(a)=\sum_{i} a_{i}(1) \gamma_{1}\left(\xi_{i}\right) .
$$

Note that $a_{i}(\lambda)$ and $a_{i}(1)$ are well-defined elements of $\mathbb{C}$ by (1.1).

Lemma 1.2. The map $\gamma$ in (1.2) is a monomorphism of $\mathbb{C}$-algebras. 
Proof. For $a=\sum_{i} a_{i}(t) \xi_{i} \in A\left(a_{i}(t) \in \mathbb{F}\right)$, let $\gamma(a)=0$. Then

$$
\sum_{i} a_{i}(\lambda) \gamma_{\lambda}\left(\xi_{i}\right)=\gamma_{\lambda}(a)=\pi_{\lambda} \gamma(a)=0
$$

for all $\lambda \in \mathbf{K}$. Hence, for each $i \in I, a_{i}(\lambda)=0$ for all $\lambda \in \mathbf{K}$ since $\left\{\gamma_{\lambda}\left(\xi_{i}\right) \mid i \in I\right\}$ is a $\mathbb{C}$-basis of $A_{\lambda}$. Thus $a_{i}(t)=0$ since $\mathbf{K}$ is an infinite set and $a_{i}(t) \in \mathbb{F}$ has finite zeros. It follows that $\gamma$ is a monomorphism.

Notation 1.3. The map

$$
\Gamma=\gamma_{1} \gamma^{-1}: \gamma(A) \longrightarrow A_{1}
$$

is a homomorphism of $\mathbb{C}$-algebras by Lemma 1.2. Note that, for $a=\sum_{i} a_{i}(t) \xi_{i} \in A$,

$$
\Gamma(\gamma(a)))=\sum_{i} a_{i}(1) \gamma_{1}\left(\xi_{i}\right)
$$

In particular, $\Gamma\left(\gamma\left(\xi_{i}\right)\right)=\gamma_{1}\left(\xi_{i}\right)$ for all $i \in I$ and thus $\Gamma$ is an epimorphism.

Theorem 1.4. Let $I$ be an ideal of $\widehat{A}$. Then $\Gamma(I \cap \gamma(A))$ is a Poisson ideal of $A_{1}$.

Proof. Since $\Gamma$ is surjective, $\Gamma(I \cap \gamma(A))$ is an ideal of $A_{1}$. It is enough to show that

$$
\left\{x^{\prime}, y^{\prime}\right\} \in \Gamma(I \cap \gamma(A))
$$

for any $x^{\prime} \in \Gamma(I \cap \gamma(A))$ and $y^{\prime} \in A_{1}$. There exist $a \in I \cap \gamma(A)$ and $b \in \gamma(A)$ such that $\gamma_{1} \gamma^{-1}(a)=x^{\prime}$ and $\gamma_{1} \gamma^{-1}(b)=y^{\prime}$. Set $\gamma^{-1}(a)=x, \gamma^{-1}(b)=y$. Thus $x^{\prime}=\gamma_{1}(x)$ and $y^{\prime}=\gamma_{1}(y)$. Since $x y-y x=(t-1) z$ for some $z \in A$ by Notation 1.1(4) and

$$
(t-1) z=x y-y x=\gamma^{-1}(a) \gamma^{-1}(b)-\gamma^{-1}(b) \gamma^{-1}(a)=\gamma^{-1}(a b-b a),
$$

we have that

$$
\gamma(t-1) \gamma(z)=\gamma((t-1) z)=a b-b a
$$

and thus

$$
\gamma(z)=[\gamma(t-1)]^{-1}(a b-b a) \in I \cap \gamma(A)
$$

since $\gamma(t-1)$ is invertible in $\widehat{A}$. Hence

$$
\left\{x^{\prime}, y^{\prime}\right\}=\left\{\gamma_{1}(x), \gamma_{1}(y)\right\}=\gamma_{1}(z) \in \Gamma(I \cap \gamma(A)),
$$

as claimed.

Corollary 1.5. Let $X$ be a subspace of the ideals of $\widehat{A}$ equipped with Zariski topology and let $Y$ be a topological subspace of $\mathrm{P}$. Spec $A_{1}$. Suppose that the map $\varphi_{\Gamma}: X \longrightarrow Y$ defined by

$$
\varphi_{\Gamma}(P)=\Gamma(P \cap \gamma(A))
$$

is a bijection satisfying the following condition: For any $P, Q \in X$, there exist subsets $I_{P}, I_{Q} \subseteq$ $\gamma(A)$ such that $I_{P}, I_{Q}, \Gamma\left(I_{P}\right)$ and $\Gamma\left(I_{Q}\right)$ generate $P, Q, \varphi_{\Gamma}(P)$ and $\varphi_{\Gamma}(Q)$, respectively, and that $I_{P} \subseteq I_{Q}$ if and only if $\Gamma\left(I_{P}\right) \subseteq \Gamma\left(I_{Q}\right)$. Then $\varphi_{\Gamma}$ is a homeomorphism from $X$ onto $Y$.

Proof. Note that $\varphi_{\Gamma}(P)$ is a Poisson ideal for any $P \in X$ by Theorem 1.4. Clearly $\varphi_{\Gamma}$ is a bijection such that $\varphi_{\Gamma}$ and $\varphi_{\Gamma}^{-1}$ preserve inclusions. Hence $\varphi_{\Gamma}$ is a homeomorphism from $X$ onto $Y$ by [․ Lemma 9.4(a)].

In the following example, we see a method how to construct a homeomorphism from $\operatorname{Spec} \mathcal{O}_{q}\left(\mathbb{C}^{2}\right)$ onto the Poisson spectrum of its semiclassical limit. 
Example 1.6. Set $\mathbf{K}=\mathbb{C} \backslash(\{0,1\} \cup\{$ roots of unity $\})$ and $\mathbb{F}=\mathbb{C}\left[t, t^{-1}\right]$. Let $A$ be the $\mathbb{F}$-algebra generated by $x, y$ subject to the relation

$$
x y=t y x .
$$

Then, for each $\lambda \in \mathbf{K}, A_{\lambda}$ is the $\mathbb{C}$-algebra generated by $x, y$ subject to the relation

$$
x y=\lambda y x
$$

and thus the set $\left\{x^{i} y^{j} \mid i, j=0,1, \ldots\right\}$ of all standard monomials forms an $\mathbb{F}$-basis of $A$ and a $\mathbb{C}$-basis of $A_{\lambda}$.

Observe that $A_{1}=A /(t-1) A$ is the Poisson $\mathbb{C}$-algebra $\mathbb{C}[x, y]$ with Poisson bracket

$$
\{x, y\}=x y \text {. }
$$

Note that the triple $(\mathbf{K}, \mathbb{F}, A)$ satisfies the conditions of Notation 1.1. We retain the notations in Notation 1.1 .

Let $0 \neq q \in \mathbb{C}$ be not a root of unity and let $\mathcal{O}_{q}\left(\mathbb{C}^{2}\right)$ be the coordinate ring of quantized affine 2 -space. That is, $\mathcal{O}_{q}\left(\mathbb{C}^{2}\right)$ is the $\mathbb{C}$-algebra generated by $x, y$ subject to the relation

$$
x y=q y x .
$$

Note that $\left\{x^{i} y^{j} \mid i, j=0,1, \ldots\right\}$ is a $\mathbb{C}$-basis of $\mathcal{O}_{q}\left(\mathbb{C}^{2}\right)$. For any $\lambda \in \mathbf{K}, \lambda$ is not a root of unity and thus there exists the $\mathbb{C}$-algebra $\mathcal{O}_{\lambda}\left(\mathbb{C}^{2}\right)$ that is defined by substituting $\lambda$ to $q$ in $\mathcal{O}_{q}\left(\mathbb{C}^{2}\right)$. (Hence $q$ is a nonzero complex number that is not a root of unity as well as plays a role as a parameter taking values in $\mathbf{K}$.) Note that $A_{\lambda}=\mathcal{O}_{\lambda}\left(\mathbb{C}^{2}\right)$ for each $\lambda \in \mathbf{K}$.

It is well-known, see [6, 2.1(ii)], that

$$
\operatorname{Spec} \mathcal{O}_{q}\left(\mathbb{C}^{2}\right)=\{\langle 0\rangle,\langle x\rangle,\langle y\rangle,\langle x-\mu, y\rangle,\langle x, y-\nu\rangle \mid \mu, \nu \in \mathbb{C}\} .
$$

Hence, for each $\lambda \in \mathbf{K}$,

$$
\operatorname{Spec} \mathcal{O}_{\lambda}\left(\mathbb{C}^{2}\right)=\{\langle 0\rangle,\langle x\rangle,\langle y\rangle,\langle x-\mu, y\rangle,\langle x, y-\nu\rangle \mid \mu, \nu \in \mathbb{C}\} .
$$

Define a map

$$
\widehat{\imath} \mathcal{O}_{q}\left(\mathbb{C}^{2}\right) \longrightarrow \widehat{A}=\prod_{\lambda \in \mathbf{K}} A_{\lambda}, \widehat{f(q)}=(f(\lambda))_{\lambda \in \mathbf{K}} .
$$

(Here $q$ is considered as a parameter taking values in $\mathbf{K}$.) Hence, for each $\lambda \in \mathbf{K}$,

$$
\pi_{\lambda}(\widehat{0})=0, \pi_{\lambda}(\widehat{x})=x, \pi_{\lambda}(\widehat{y})=y, \pi_{\lambda}(\widehat{x-\mu})=x-\mu, \pi_{\lambda}(\widehat{y-\nu})=y-\nu .
$$

Note that

$$
\gamma(0)=\widehat{0}, \quad \gamma(x)=\widehat{x}, \quad \gamma(y)=\widehat{y}, \quad \gamma(x-\mu)=\widehat{x-\mu}, \quad \gamma(y-\nu)=\widehat{y-\nu} .
$$

Let $X$ be the set consisting of the following ideals of $\widehat{A}$

$$
X=\{\langle\widehat{0}\rangle,\langle\widehat{x}\rangle,\langle\widehat{y}\rangle,\langle\widehat{x-\mu}, \widehat{y}\rangle,\langle\widehat{x}, \widehat{y-\nu}\rangle \mid \mu, \nu \in \mathbb{C}\} .
$$

The map (1.6) is an injective map preserving inclusions since $q \in \mathbf{K}$ and thus (1.6) induces a canonical homeomorphism $\widehat{\varphi}$ from $\operatorname{Spec} \mathcal{O}_{q}\left(\mathbb{C}^{2}\right)$ onto $X$. Note that all ideals in $X$ are generated by elements of $\gamma(A)$, that

$$
\varphi_{\Gamma}(X)=\{\langle 0\rangle,\langle x\rangle,\langle y\rangle,\langle x-\mu, y\rangle,\langle x, y-\nu\rangle \mid \mu, \nu \in \mathbb{C}\}
$$


is equal to P.Spec $A_{1}$ by [5, 9.5] and that $\varphi_{\Gamma}$ is a homeomorphism from $X$ onto $\varphi_{\Gamma}(X)$ by Corollary 1.5. Hence the composition $\varphi_{\Gamma} \circ \widehat{\varphi}$ is a homeomorphism from $\operatorname{Spec} \mathcal{O}_{q}\left(\mathbb{C}^{2}\right)$ onto P. Spec $A_{1}$.

\section{Multi-Parameter Poisson Weyl algebras}

Definition 2.1. (See [9].) Let $Q=\left(q_{1}, \ldots, q_{n}\right)$ be an $n$-tuple of elements of $\mathbb{C} \backslash\{0,1\}$ and let $\Lambda=\left(\lambda_{i j}\right)$ be a multiplicative antisymmetric $n \times n$-matrix over $\mathbb{C} \backslash\{0\}$, namely $\lambda_{i j}=\lambda_{j i}^{-1}$ for $i \neq j$ and $\lambda_{i i}=1$. The multi-parameter quantized Weyl algebra $R_{n}^{Q, \Lambda}$ is the $\mathbb{C}$-algebra generated by $y_{1}, x_{1}, \ldots, y_{n}, x_{n}$ subject to the relations

$$
\begin{array}{rlrl}
y_{j} y_{i} & =\lambda_{j i} y_{i} y_{j} & & 1 \leq i<j \leq n \\
y_{j} x_{i} & =\lambda_{i j} x_{i} y_{j} & & 1 \leq i<j \leq n \\
x_{j} y_{i} & =q_{i} \lambda_{i j} y_{i} x_{j} & 1 \leq i<j \leq n \\
x_{j} x_{i} & =q_{i}^{-1} \lambda_{i j}^{-1} x_{i} x_{j} & 1 \leq i<j \leq n \\
x_{i} y_{i}-q_{i} y_{i} x_{i} & =1+\sum_{k=1}^{i-1}\left(q_{k}-1\right) y_{k} x_{k} & & 1 \leq i \leq n .
\end{array}
$$

Notation 2.2. (1) Denote by $G(Q, \Lambda)$ the multiplicative subgroup of $\mathbb{C} \backslash\{0\}$ generated by all $q_{i}$ and $\lambda_{i j}, 1 \leq i, j \leq n$.

(2) Assume throughout the section that $G(Q, \Lambda)$ is torsion free. Hence $G(Q, \Lambda)$ is a free abelian group with finite rank, say $r$. Fix a basis $\left\{\eta_{1}, \ldots, \eta_{r}\right\}$ of $G(Q, \Lambda)$, a $\mathbb{Q}$-linearly independent subset $\left\{\mu_{1}, \ldots, \mu_{r}\right\}$ of $\mathbb{C}$ and a non-root of unity $q \in \mathbb{C} \backslash\{0,1\}$.

(3) For each $i=1, \ldots, r$, there exists a unique element $e_{i}=a_{i} t^{2}+b_{i} t+c_{i} \in \mathbb{C}[t]$ such that

$$
e_{i}(q)=\eta_{i}, e_{i}(1)=1, e_{i}^{\prime}(1)=\mu_{i},
$$

where $e_{i}^{\prime}$ is the formal derivative of $e_{i}$, since the determinant of the matrix $\left(\begin{array}{lll}q^{2} & q & 1 \\ 1 & 1 & 1 \\ 2 & 1 & 0\end{array}\right)$ is nonzero.

(3) Let $\mathbb{F}$ be the ring

$$
\mathbb{F}=\mathbb{C}\left[t, t^{-1}\right]\left[e_{1}^{-1}, \ldots, e_{r}^{-1}\right] .
$$

and let $U(\mathbb{F})$ be the unit group of $\mathbb{F}$. For any $c \in G(Q, \Lambda)$, there exists a unique element $\left(s_{1}, \ldots, s_{r}\right) \in \mathbb{Z}^{r}$ such that $c=\eta_{1}^{s_{1}} \eta_{2}^{s_{2}} \cdots \eta_{r}^{s_{r}}$. Define a homomorphism of groups

$$
\sim: G(Q, \Lambda) \longrightarrow U(\mathbb{F}), \quad \widetilde{c}=e_{1}^{s_{1}} e_{2}^{s_{2}} \cdots e_{r}^{s_{r}} .
$$

If $\widetilde{c}=1$ then $c=\widetilde{c}(q)=1$ and thus $\sim$ is a monomorphism of groups such that $\widetilde{\eta}_{i}=e_{i}$ for all $i=1, \ldots, r$.

Definition 2.3. Set

$$
\widetilde{Q}=\left(\widetilde{q}_{1}, \ldots, \widetilde{q}_{n}\right), \quad \widetilde{\Lambda}=\left(\widetilde{\lambda}_{i j}\right) .
$$


Note that $\widetilde{q}_{i} \neq 1$ and that $\widetilde{\Lambda}$ is a multiplicative antisymmetric $n \times n$-matrix. Define $A_{n}^{\widetilde{Q}, \widetilde{\Lambda}}$ to be the $\mathbb{F}$-algebra generated by $y_{1}, x_{1}, \ldots, y_{n}, x_{n}$ subject to the relations

$$
\begin{array}{rlrl}
y_{j} y_{i} & =\widetilde{\lambda}_{j i} y_{i} y_{j} & 1 \leq i<j \leq n \\
y_{j} x_{i} & =\widetilde{\lambda}_{i j} x_{i} y_{j} & 1 \leq i<j \leq n \\
x_{j} y_{i} & =\widetilde{q}_{i} \widetilde{\lambda}_{i j} y_{i} x_{j} & 1 \leq i<j \leq n \\
x_{j} x_{i} & =\widetilde{q}_{i}^{-1} \widetilde{\lambda}_{i j}^{-1} x_{i} x_{j} & 1 \leq i<j \leq n \\
x_{i} y_{i}-\widetilde{q}_{i} y_{i} x_{i} & =\left(\widetilde{q}_{i}-1\right)\left(1+\sum_{k=1}^{i-1} y_{k} x_{k}\right) & & 1 \leq i \leq n .
\end{array}
$$

Write $A$ for $A_{n}^{\widetilde{Q}, \widetilde{\Lambda}}$ for simplicity.

Lemma 2.4. The algebra $A$ is the iterated skew polynomial algebra

$$
A=\mathbb{F}\left[y_{1}\right]\left[x_{1} ; \beta^{1}, \delta^{1}\right]\left[y_{2} ; \alpha^{2}\right]\left[x_{2} ; \beta^{2}, \delta^{2}\right] \ldots\left[y_{n} ; \alpha^{n}\right]\left[x_{n} ; \beta^{n}, \delta^{n}\right],
$$

where

$$
\begin{aligned}
& \alpha^{j}\left(y_{i}\right)=\widetilde{\lambda}_{j i} y_{i} \\
& i<j, \quad \alpha^{j}\left(x_{i}\right)=\widetilde{\lambda}_{i j} x_{i} \quad i<j \\
& \beta^{j}\left(y_{i}\right)=\widetilde{q}_{i} \widetilde{\lambda}_{i j} y_{i} \\
& i \leq j \text {, } \\
& \beta^{j}\left(x_{i}\right)=\widetilde{q}_{i}^{-1} \widetilde{\lambda}_{i j}^{-1} x_{i} \quad i<j \\
& \delta^{j}\left(y_{i}\right)=0 \\
& i<j \text {, } \\
& \delta^{j}\left(x_{i}\right)=0 \\
& i<j \\
& \delta^{j}\left(y_{j}\right)=\left(\widetilde{q}_{j}-1\right)\left(1+\sum_{k=1}^{j-1} y_{k} x_{k}\right) \quad 1 \leq j \leq n .
\end{aligned}
$$

Hence $\left\{y_{1}^{r_{1}} x_{1}^{s_{1}} \cdots y_{n}^{r_{n}} x_{n}^{s_{n}} \mid r_{i}, s_{i}=0,1, \ldots\right\}$ forms an $\mathbb{F}$-basis of $A$.

Proof. It is verified by (2.4).

Lemma 2.5. Let $\mathbf{K}$ be the set consisting of $\lambda \in \mathbb{C} \backslash\{0,1\}$ such that $e_{i}(\lambda) \neq 0$ for all $1 \leq i \leq r$ and the multiplicative subgroup $\left\langle e_{1}(\lambda), \ldots, e_{r}(\lambda)\right\rangle$ is a free abelian group with basis $e_{1}(\lambda), \ldots, e_{r}(\lambda)$. Then $\mathbf{K}$ is an infinite subset of $\mathbb{C} \backslash\{0,1\}$ and $q \in \mathbf{K}$.

Proof. Let $f_{1}(t), \ldots, f_{r}(t)$ be nonconstant and continuous functions on a non-empty open set $U \subseteq \mathbb{C} \backslash\{0,1\}$ such that $f_{i}(t) / f_{j}(t)$ is nonconstant for each pair $(i, j)$ with $i \neq j$. We show that there are uncountably many $0,1 \neq \lambda \in \mathbb{C}$ such that $\left\{f_{i}(\lambda)\right\}$ is $\mathbb{Q}$-linearly independent. We proceed by using induction on $r$. If $r=1$ then it is true clearly since $f_{1}$ is a nonconstant and continuous function. Suppose that $r>1$ and that the claim is true for $r-1$. By the induction hypothesis, there are uncountably many $0,1 \neq \lambda \in \mathbb{C}$ such that $\sum_{i=1}^{r-1} a_{i}\left(f_{i} / f_{r}\right)(\lambda) \neq$ 0 for any $0 \neq\left(a_{1}, \ldots, a_{r-1}\right) \in \mathbb{Q}^{r-1}$. Hence, for any $a_{r} \in \mathbb{Q}$ such that $0 \neq\left(a_{1}, \ldots, a_{r}\right)$, $\sum_{i=1}^{r-1} a_{i}\left(f_{i} / f_{r}\right)(\lambda)+a_{r} \neq 0$ for uncountably many $0,1 \neq \lambda \in \mathbb{C}$ since the cardinality of $\mathbb{Q}$ is countable. It follows that $\sum_{i=1}^{r} a_{i} f_{i}(\lambda) \neq 0$ for any $0 \neq\left(a_{1}, \ldots, a_{r}\right) \in \mathbb{Q}^{r}$ and thus the claim is verified.

Let $\ell$ be a half line starting from the origin such that $e_{i}(q) \notin \ell$ for all $i=1, \ldots, r$. Set $f_{i}(t)=\log e_{i}(t)$ for $i=1, \ldots, r$, where the function log is defined in the branch determined by $\ell$. Then all $f_{i}(t)$ are nonconstant and continuous on a nonempty open set $q \in U \subseteq \mathbb{C} \backslash\{0,1\}$ such 
that each $e_{i}(U)$ is contained in the given branch since $e_{i}(t)$ is a nonconstant polynomial by (2.2). For each pair $(i, j)$ with $i \neq j$, suppose that $f_{i}(t) / f_{j}(t)=c \in \mathbb{Q}$. Then $\eta_{i}=e_{i}(q)=e_{j}^{c}(q)=\eta_{j}^{c}$, which is impossible since $\left\{\eta_{k}\right\}_{k=1}^{r}$ is a basis of $G(Q, \Lambda)$. If $f_{i}(t) / f_{j}(t)=c \in \mathbb{C} \backslash \mathbb{Q}$ then $e_{i}=e_{j}^{c}$, a contradiction since all $e_{i}$ are polynomials. Hence, for each pair $(i, j)$ with $i \neq j, f_{i}(t) / f_{j}(t)$ is not constant. Therefore there are uncountably many $0,1 \neq \lambda \in \mathbb{C}$ such that $\left\{f_{i}(\lambda)\right\}$ is $\mathbb{Q}$-linearly independent by the claim. This completes the proof.

We apply Notation 1.1 to the triple $(\mathbf{K}, \mathbb{F}, A)$. Note that $(\mathbf{K}, \mathbb{F}, A)$ satisfies Notation 1.1(1)-(3).

Notation 2.6. Note that $t-1$ is a nonzero, nonunit and non-zero-divisor of $A$. Set

$$
A_{1}=A /(t-1) A \text {. }
$$

It is proved by the proof of Theorem $2.9(3)$ that $A_{1}$ is a commutative $\mathbb{C}$-algebra. Thus $(\mathbf{K}, \mathbb{F}, A)$ satisfies the condition of Notation 1.1(4).

For each $\lambda \in \mathbf{K}$, the $\mathbb{C}$-algebra $A_{\lambda}$ is defined by Notation 1.1 ( 5$)$.

Proposition 2.7. (1) For each $\lambda \in \mathbf{K}, A_{\lambda}$ is the $\mathbb{C}$-algebra generated by $y_{1}, x_{1}, \ldots, y_{n}, x_{n}$ subject to the relations

$$
\begin{array}{rlrl}
y_{j} y_{i} & =\widetilde{\lambda}_{j i}(\lambda) y_{i} y_{j} & 1 \leq i<j \leq n \\
y_{j} x_{i} & =\widetilde{\lambda}_{i j}(\lambda) x_{i} y_{j} & 1 \leq i<j \leq n \\
x_{j} y_{i} & =\widetilde{q}_{i}(\lambda) \widetilde{\lambda}_{i j}(\lambda) y_{i} x_{j} & 1 \leq i<j \leq n \\
x_{j} x_{i} & =\widetilde{q}_{i}^{-1}(\lambda) \widetilde{\lambda}_{i j}^{-1}(\lambda) x_{i} x_{j} & 1 \leq i<j \leq n \\
x_{i} y_{i}-\widetilde{q}_{i}(\lambda) y_{i} x_{i} & =\left(\widetilde{q}_{i}(\lambda)-1\right)\left(1+\sum_{k=1}^{i-1} y_{k} x_{k}\right) & & 1 \leq i \leq n
\end{array}
$$

and an iterated skew polynomial algebra

$$
A_{\lambda}=\mathbb{C}\left[y_{1}\right]\left[x_{1} ; \beta_{\lambda}^{1}, \delta_{\lambda}^{1}\right]\left[y_{2} ; \alpha_{\lambda}^{2}\right]\left[x_{2} ; \beta_{\lambda}^{2}, \delta_{\lambda}^{2}\right] \cdots\left[y_{n} ; \alpha_{\lambda}^{n}\right]\left[x_{n} ; \beta_{\lambda}^{n}, \delta_{\lambda}^{n}\right]
$$

for suitable $\alpha_{\lambda}^{i}, \beta_{\lambda}^{i}, \delta_{\lambda}^{i}$. Hence $\left\{y_{1}^{r_{1}} x_{1}^{s_{1}} \cdots y_{n}^{r_{n}} x_{n}^{s_{n}} \mid r_{i}, s_{i}=0,1, \ldots\right\}$ forms a $\mathbb{C}$-basis of $A_{\lambda}$.

(2) In particular, $A_{q}$ is the $\mathbb{C}$-algebra generated by $y_{1}, x_{1}, \ldots, y_{n}, x_{n}$ subject to the relations

$$
\begin{array}{rlrl}
y_{j} y_{i} & =\lambda_{j i} y_{i} y_{j} & 1 \leq i<j \leq n \\
y_{j} x_{i} & =\lambda_{i j} x_{i} y_{j} & 1 \leq i<j \leq n \\
x_{j} y_{i} & =q_{i} \lambda_{i j} y_{i} x_{j} & 1 \leq i<j \leq n \\
x_{j} x_{i} & =q_{i}^{-1} \lambda_{i j}^{-1} x_{i} x_{j} & 1 \leq i<j \leq n \\
x_{i} y_{i}-q_{i} y_{i} x_{i} & =\left(q_{i}-1\right)\left(1+\sum_{k=1}^{i-1} y_{k} x_{k}\right) & & 1 \leq i \leq n .
\end{array}
$$

Proof. (1) It follows from (2.4) and (2.5).

(2) Since $q \in \mathbf{K}$ and $e_{i}(q)=\eta_{i}$ for all $i$,

$$
\widetilde{\lambda}_{i j}(q)=\lambda_{i j}, \quad \widetilde{q}_{i}(q)=q_{i}
$$

for all $i, j$. Hence the result follows from (2.6) by replacing $\lambda$ by $q$. 
Lemma 2.8. The multi-parameter quantized Weyl algebra $R_{n}^{Q, \Lambda}$ is isomorphic to $A_{q}$.

Proof. Define a homomorphism of $\mathbb{C}$-algebras

$$
\psi: R_{n}^{Q, \Lambda} \longrightarrow A_{q} \psi\left(x_{i}\right)=x_{i}, \psi\left(y_{i}\right)=\left(q_{i}-1\right)^{-1} y_{i}
$$

for all $i$. Then $\psi$ is an isomorphism by (2.1) and (2.7).

Theorem 2.9. Let $G(Q, \Lambda)$ be torsion free.

(1) $\widetilde{Q}^{\prime}(1):=\left(\widetilde{q}_{1}^{\prime}(1), \ldots, \widetilde{q}_{n}^{\prime}(1)\right)$ is an $n$-tuple of elements of $\mathbb{C} \backslash\{0\}$.

(2) $\widetilde{\Lambda}^{\prime}(1):=\left(\widetilde{\lambda}_{i j}^{\prime}(1)\right)$ is a skew-symmetric $n \times n$-matrix over $\mathbb{C}$.

(3) The $\mathbb{C}$-algebra $A_{1}$ is the Poisson algebra $\mathbb{C}\left[y_{1}, x_{1}, \ldots, y_{n}, x_{n}\right]$ with Poisson bracket

$$
\begin{aligned}
& \left\{y_{j}, y_{i}\right\}=\widetilde{\lambda}_{j i}^{\prime}(1) y_{i} y_{j} \quad 1 \leq i<j \leq n \\
& \left\{y_{j}, x_{i}\right\}=\widetilde{\lambda}_{i j}^{\prime}(1) x_{i} y_{j} \quad 1 \leq i<j \leq n \\
& \left\{x_{j}, y_{i}\right\}=\left(\widetilde{q}_{i}^{\prime}(1)+\widetilde{\lambda}_{i j}^{\prime}(1)\right) y_{i} x_{j} \quad 1 \leq i<j \leq n \\
& \left\{x_{j}, x_{i}\right\}=-\left(\widetilde{q}_{i}^{\prime}(1)+\widetilde{\lambda}_{i j}^{\prime}(1)\right) x_{i} x_{j} \quad 1 \leq i<j \leq n \\
& \left\{x_{i}, y_{i}\right\}=\widetilde{q}_{i}^{\prime}(1)\left(1+\sum_{k=1}^{i} y_{k} x_{k}\right) \quad 1 \leq i \leq n,
\end{aligned}
$$

where $\widetilde{q}_{i}^{\prime}, \widetilde{\lambda}_{i j}^{\prime}$ are the formal derivatives of $\widetilde{q}_{i}, \widetilde{\lambda}_{i j}$ respectively. We will call $A_{1}$ the multi-parameter Poisson Weyl algebra.

Remark 2.10. Note that $(\mathbf{K}, \mathbb{F}, A)$ satisfies the condition of Notation 1.1(7) by Lemma 2.4, Proposition 2.7(1) and Theorem 2.9)(3). We retain the notations in Notation 1.1 for $(\mathbf{K}, \mathbb{F}, A)$.

Proof of Theorem [2.9. (1) Let $q_{i}=\eta_{1}^{s_{1 i}} \eta_{2}^{s_{2 i}} \cdots \eta_{r}^{s_{r i}}$. Since $q_{i} \neq 1, s_{k i} \neq 0$ for some $k$. Thus, by (2.2),

$$
\widetilde{q}_{i}^{\prime}(1)=s_{1 i} \mu_{1}+\ldots+s_{r i} \mu_{r} \neq 0 .
$$

(2) For all $1 \leq i, j \leq n$, since $\lambda_{i j} \lambda_{j i}=1, \widetilde{\lambda}_{i j} \widetilde{\lambda}_{j i}=1$ by (2.3) and thus $\widetilde{\lambda}_{i j}^{\prime}(1)+\widetilde{\lambda}_{j i}^{\prime}(1)=0$ by (2.2).

(3) Since $e_{i}(1)=1$ by (2.2) $, \widetilde{q}_{i}(1)=1, \widetilde{\lambda}_{i j}(1)=1$ for all $i, j$ and thus $A_{1}$ is commutative by (2.4). Let us find the Poisson bracket in $A_{1}$. For $i<j$,

$$
\left\{y_{j}, y_{i}\right\}=\left.(t-1)^{-1}\left(y_{j} y_{i}-y_{i} y_{j}\right)\right|_{t=1}=\left(\frac{\widetilde{\lambda}_{j i}-1}{t-1}\right)(1) y_{i} y_{j}=\widetilde{\lambda}_{j i}^{\prime}(1) y_{i} y_{j}
$$

and thus the first formula of (2.9) is obtained. The others of (2.9) are obtained similarly.

Notation 2.11. In $A_{q}$ and $A_{1}$, set

$$
z_{0}=1, \quad z_{i}=1+\sum_{k=1}^{i} y_{k} x_{k}
$$

for $i=1, \ldots, n$. 
Lemma 2.12. (1) In $A_{q}$,

$$
x_{i} y_{i}-q_{i} y_{i} x_{i}=\left(q_{i}-1\right) z_{i-1}, \quad x_{i} y_{i}-y_{i} x_{i}=\left(q_{i}-1\right) z_{i}, \quad 1 \leq i \leq n
$$

and

(2) In $A_{1}$,

$$
\begin{array}{llll}
y_{j} z_{i}=z_{i} y_{j} & i<j ; \quad x_{j} z_{i}=z_{i} x_{j} & i<j \\
y_{j} z_{i}=q_{j}^{-1} z_{i} y_{j} & i \geq j ; & x_{j} z_{i}=q_{j} z_{i} x_{j} & i \geq j \\
z_{i} z_{j}=z_{j} z_{i} & \text { all } i, j . & &
\end{array}
$$

$$
\left\{x_{i}, y_{i}\right\}-\widetilde{q}_{i}^{\prime}(1) y_{i} x_{i}=\widetilde{q}_{i}^{\prime}(1) z_{i-1}, \quad\left\{x_{i}, y_{i}\right\}=\widetilde{q}_{i}^{\prime}(1) z_{i}, \quad 1 \leq i \leq n
$$

and

$$
\begin{array}{llll}
\left\{y_{j}, z_{i}\right\}=0 & i<j ; & \left\{x_{j}, z_{i}\right\}=0 & i<j ; \\
\left\{y_{j}, z_{i}\right\}=-\widetilde{q}_{j}^{\prime}(1) y_{j} z_{i} & i \geq j ; & \left\{x_{j}, z_{i}\right\}=\widetilde{q}_{j}^{\prime}(1) x_{j} z_{i} & i \geq j ; \\
\left\{z_{i}, z_{j}\right\}=0 & \text { all } i, j . & &
\end{array}
$$

Proof. These are checked routinely by (2.7) and (2.9).

Definition 2.13. A subset $T$ of $\mathcal{M}_{n}=\left\{z_{1}, z_{2}, y_{2}, x_{2}, z_{3}, y_{3}, x_{3}, \ldots, z_{n}, y_{n}, x_{n}\right\}$ is said to be admissible if $T$ satisfies the condition: For $2 \leq i \leq n$,

$$
y_{i} \in T \text { or } x_{i} \in T \Leftrightarrow z_{i} \in T \text { and } z_{i-1} \in T \text {. }
$$

Note that if $T$ is an admissible set of $A_{q}$ then the ideal $\langle T\rangle$ generated by $T$ is equal to the right (left) ideal generated by $T$ since each $z_{i}$ is normal and $y_{i}$ and $x_{i}$ are normal modulo $\left\langle z_{i-1}\right\rangle$ by Lemma $2.12(1)$.

Lemma 2.14. (1) Let $P$ be a prime ideal of $A_{q}$. Then $P \cap \mathcal{M}_{n}$ is an admissible set.

(2) Let $P$ be a Poisson prime ideal of $A_{1}$. Then $P \cap \mathcal{M}_{n}$ is an admissible set.

Proof. (1) If $y_{i} \in P \cap \mathcal{M}_{n}$ or $x_{i} \in P \cap \mathcal{M}_{n}$ then $z_{i}, z_{i-1} \in P \cap \mathcal{M}_{n}$ by Lemma2.12(1). Conversely, if $z_{i}, z_{i-1} \in P \cap \mathcal{M}_{n}$ then $y_{i} x_{i}=z_{i}-z_{i-1} \in P$. Since $y_{i}$ and $x_{i}$ are normal modulo $\left\langle z_{i-1}\right\rangle$, $y_{i} \in P \cap \mathcal{M}_{n}$ or $x_{i} \in P \cap \mathcal{M}_{n}$.

(2) Similar to (1).

Corollary 2.15. For each admissible set $T$, set

$$
\begin{aligned}
\operatorname{Spec}_{T} A_{q} & =\left\{P \in \operatorname{Spec} A_{q} \mid P \cap \mathcal{M}_{n}=T\right\}, \\
\text { P. } \operatorname{Spec}_{T} A_{1} & =\left\{P \in \mathrm{P} . \operatorname{Spec} A_{1} \mid P \cap \mathcal{M}_{n}=T\right\} .
\end{aligned}
$$

Then

$$
\operatorname{Spec} A_{q}=\biguplus_{T} \operatorname{Spec}_{T} A_{q}, \quad \text { P. Spec } A_{1}=\biguplus_{T} \text { P. } \operatorname{Spec}_{T} A_{1} .
$$

Proof. It is clear by Lemma 2.14

Remark 2.16. Recall the following concepts in [1, 4.2]. In $R_{n}^{Q, \Lambda}$, set $z_{i}=1+\sum_{k=1}^{i}\left(q_{k}-1\right) y_{k} x_{k}$ for each $1 \leq i \leq n$. For a subset $U$ of $\left\{z_{1}, y_{1}, x_{1}, z_{2}, y_{2}, x_{2}, \ldots, z_{n}, y_{n}, x_{n}\right\} \subseteq R_{n}^{Q, \Lambda}$ and an integer $i$ with $1 \leq i \leq n$, we say that $i$ features in $U$ if at least one of $z_{i}, y_{i}, x_{i}$ is in $U$. By $p$-sequence, we mean a subset $U$ of $\left\{z_{1}, y_{1}, x_{1}, \ldots, z_{n}, y_{n}, x_{n}\right\}$ with the following properties:

(i) if $z_{i} \in U$ then $i-1$ does not feature in $U$;

(ii) if $y_{i} \in U$ or $x_{i} \in U$ then $i-1$ does feature in $U$. 
Let $U$ be a $p$-sequence such that $y_{1}, x_{1} \notin U$ and set $U^{\prime}=U \cup\left\{z_{i} \mid i\right.$ features in $\left.U\right\}$. Then the homomorphic image $\psi\left(U^{\prime}\right)$, where $\psi$ is the isomorphism (2.8), consists of elements obtained by multiplying nonzero scalars to all elements in an admissible set $T$. Thus the ideal generated by $\psi(U)$ is equal to the ideal $\langle T\rangle$. Conversely, for any admissible set $T$ of $A_{q},\langle T\rangle$ is equal to an ideal generated by $\psi(U)$ for some $p$-sequence $U$ such that $y_{1}, x_{1} \notin U$.

Lemma 2.17. Let $G(Q, \Lambda)$ be torsion free and let $T$ be an admissible set.

(1) The ideal $\langle T\rangle$ of $A_{q}$ is a completely prime ideal.

(2) The ideal $\langle T\rangle$ of $A_{1}$ is a Poisson prime ideal.

Proof. (1) It follows immediately by Remark 2.16 and [1, 4.5].

(2) We proceed by induction on $n$. If $n=1$ then the admissible sets of $\mathcal{M}_{1}$ are only $\varnothing$ and $\left\{z_{1}\right\}$. If $T=\varnothing$ then $\langle T\rangle=0$ and thus the result is clear. Let $T=\left\{z_{1}\right\}$. Since $z_{1}$ is a Poisson normal element, $\langle T\rangle$ is Poisson prime.

Suppose that $n>1$ and that the result is true for the cases less than $n$. Set $U=T \cap \mathcal{M}_{n-1}$. Then $U$ is an admissible set of $\mathcal{M}_{n-1}$. There are following five cases:

$$
T=U, T=U \cup\left\{z_{n}\right\}, T=U \cup\left\{z_{n}, y_{n}\right\}, T=U \cup\left\{z_{n}, x_{n}\right\}, T=U \cup\left\{z_{n}, y_{n}, x_{n}\right\} .
$$

If $T=U$ then the result is clear by the induction hypothesis. For the other cases, $\langle T\rangle$ is Poisson prime since $z_{n}$ is Poisson normal and $y_{n}$ and $x_{n}$ are Poisson normal modulo $\left\langle z_{n}\right\rangle$.

Notation 2.18. Let $T$ be an admissible set of $\mathcal{M}_{n}$. Define a subset $Y_{T}$ of $\mathcal{M}_{n} \cup\left\{y_{1}\right\}$ by

$$
Y_{T} \cap\left\{z_{i}, y_{i}, x_{i}\right\}= \begin{cases}\left\{z_{i}, y_{i}\right\} & \text { if } z_{i} \notin T, \\ \left\{y_{i}\right\} & \text { if } z_{i} \in T, y_{i} \notin T, \\ \left\{x_{i}\right\} & \text { if } z_{i} \in T, y_{i} \in T, x_{i} \notin T, \\ \varnothing & \text { if } z_{i} \in T, y_{i} \in T, x_{i} \in T\end{cases}
$$

for each $i=1, \ldots, n$. Note that there is no $i$ such that $y_{i} \in Y_{T}$ and $x_{i} \in Y_{T}$.

Recall the coordinate ring of quantum torus: Let $C=\left(c_{i j}\right)$ be a multiplicative antisymmetric $n \times n$-matrix over $\mathbb{C}$. The coordinate ring of quantum torus, denoted by $\mathcal{T}_{C}$, is the $\mathbb{C}$-algebra generated by $z_{1}^{ \pm 1}, \ldots, z_{n}^{ \pm 1}$ subject to the relations

$$
z_{i} z_{j}=c_{i j} z_{j} z_{i}
$$

for all $1 \leq i, j \leq n$.

The proof of the following lemma is modified from that of [1, 4.9] by using Poisson terminologies.

Lemma 2.19. Let $T$ be an admissible set of $\mathcal{M}_{n}$ and set $Y_{T}=\left\{w_{1}, \ldots, w_{s}\right\}$. Denote by $\mathcal{Y}_{T}$ the multiplicative closed set of $A_{q} /\langle T\rangle$ and $A_{1} /\langle T\rangle$ generated by the canonical image of $Y_{T}$.

(1) Let $\mathbb{C}\left[w_{1}, \ldots, w_{s}\right]$ be the subalgebra of $A_{q}$ generated by $w_{1}, \ldots, w_{s}$. Then, for all $1 \leq i, j \leq$ $s$,

$$
w_{i} w_{j}=c_{i j} w_{j} w_{i}
$$

for some $c_{i j} \in G(Q, \Lambda)$ and $\left(A_{q} /\langle T\rangle\right)\left[\mathcal{Y}_{T}^{-1}\right]$ is isomorphic to the localization $\mathbb{C}\left[w_{1}^{ \pm 1}, \ldots, w_{s}^{ \pm 1}\right]$ of $\mathbb{C}\left[w_{1}, \ldots, w_{s}\right]$ at the multiplicative closed set generated by $w_{1}, \ldots, w_{s}$, which is the coordinate ring of quantum torus $\mathcal{T}_{C_{T}}$, where $C_{T}=\left(c_{i j}\right)$ a multiplicative anti-symmetric $s \times$ s-matrix. 
(2) Let $\mathbb{C}\left[w_{1}, \ldots, w_{s}\right]$ be the Poisson subalgebra of $A_{1}$ generated by $w_{1}, \ldots, w_{s}$. Then, for all $1 \leq i, j \leq s$,

$$
\left\{w_{i}, w_{j}\right\}=\widetilde{c}_{i j}(1) w_{i} w_{j}
$$

where $c_{i j}$ is the element of $G(Q, \Lambda)$ in (1), and $\left(A_{1} /\langle T\rangle\right)\left[\mathcal{Y}_{T}^{-1}\right]$ is Poisson isomorphic to the localization $\mathbb{C}\left[w_{1}^{ \pm 1}, \ldots, w_{s}^{ \pm 1}\right]$ of $\mathbb{C}\left[w_{1}, \ldots, w_{s}\right]$ at the multiplicative closed set generated by $w_{1}, \ldots, w_{s}$.

Proof. (1) There is no $i$ such that $y_{i} \in Y_{T}$ and $x_{i} \in Y_{T}$. Hence, for all $1 \leq i, j \leq s, w_{i} w_{j}=c_{i j} w_{j} w_{i}$ for a suitable element $c_{i j} \in G(Q, \Lambda)$ by (2.7) and Lemma 2.12(1). The remaining result is proved by [1, 4.8 and the proof of 4.9] since $Y_{T}$ consists of the elements obtained by multiplying nonzero scalars to the elements of $\psi(D(U))$ for some $p$-sequence $U$ by Remark 2.16, where $\psi$ is the isomorphism (2.8) and $D(U)$ is the subset of $R_{n}^{Q, \Lambda}$ in [1, 4.8].

(2) Note that the canonical map from the subalgebra of $A_{1}$ generated by $Y_{T}$ to $A_{1} /\langle T\rangle$ is injective and that, for $i$ with $z_{i} \notin T, y_{i}$ and $z_{i}$ are in $Y_{T}$ and $\bar{x}_{i}=\bar{y}_{i}^{-1} \overline{\left(z_{i}-z_{i-1}\right)}$ in $\left(A_{1} /\langle T\rangle\right)\left[\mathcal{Y}_{T}^{-1}\right]$. Hence $\left(A_{1} /\langle T\rangle\right)\left[\mathcal{Y}_{T}^{-1}\right]$ is the Poisson algebra $\mathbb{C}\left[w_{1}^{ \pm 1}, \ldots, w_{s}^{ \pm 1}\right]$.

It remains to prove the Poisson bracket $\left\{w_{i}, w_{j}\right\}=\widetilde{c}_{i j}(1) w_{i} w_{j}$ for all $1 \leq i, j \leq s$. We proceed by induction on $n$. If $n=1$ then $Y_{T}=\left\{z_{1}, y_{1}\right\}$ or $Y_{T}=\left\{y_{1}\right\}$. If $Y_{T}=\left\{z_{1}, y_{1}\right\}$ then $z_{1} y_{1}=q_{1} y_{1} z_{1}$ in $A_{q}$ and $\left\{z_{1}, y_{1}\right\}=\widetilde{q}_{1}^{\prime}(1) z_{1} y_{1}$ in $A_{1}$ by Lemma 2.12. If $Y_{T}=\left\{y_{1}\right\}$ then $y_{1} y_{1}=y_{1} y_{1}$ in $A_{q}$ and $\left\{y_{1}, y_{1}\right\}=0$ in $A_{1}$. Hence our claim is true for $n=1$.

Suppose that $n>1$ and that our claim is true for the cases less than $n$. Let $W=T \cap \mathcal{M}_{n-1}$. Then $W$ is an admissible set of $\mathcal{M}_{n-1}$. Set $Y_{W}=\left\{w_{1}, \ldots, w_{p}\right\}$. Let $\Delta$ be the multiplicative antisymmetric $p \times p$-matrix $\left(c_{i j}\right)$ which is determined by the commutation relations $w_{i} w_{j}=c_{i j} w_{j} w_{i}$ for all $1 \leq i, j \leq p$ and let $\Delta^{\prime}$ be the skew symmetric $p \times p$-matrix $\left(d_{i j}\right)$ which is determined by the Poisson brackets $\left\{w_{i}, w_{j}\right\}=d_{i j} w_{i} w_{j}$ for all $1 \leq i, j \leq p$. By induction hypothesis, $d_{i j}=\widetilde{c}_{i j}(1)$ for all $1 \leq i, j \leq p$. There are the following five cases:

$$
T=W, T=W \cup\left\{z_{n}\right\}, T=W \cup\left\{z_{n}, y_{n}\right\}, T=W \cup\left\{z_{n}, x_{n}\right\}, T=W \cup\left\{z_{n}, y_{n}, x_{n}\right\} .
$$

If $T=W$ then $Y_{T}=Y_{W} \cup\left\{z_{n}, y_{n}\right\}=\left\{w_{1}, \ldots, w_{p}, z_{n}, y_{n}\right\}$. In $A_{q}$, the commutation relations of each pair of elements in $Y_{T}$ are determined by the multiplicative anti-symmetric matrix:

$$
\left(\begin{array}{cc}
\Delta & U \\
U^{*} & V
\end{array}\right)
$$

where $U$ is the $p \times 2$-matrix determined by the commutation relations between $\left\{w_{1}, \ldots, w_{p}\right\}$ and $\left\{z_{n}, y_{n}\right\}$, each $(k, \ell)$-entry of $U^{*}$ is the inverse of the $(\ell, k)$-entry of $U$ and $V$ is the multiplicative anti-symmetric matrix $\left(\begin{array}{cc}1 & q_{n} \\ q_{n}^{-1} & 1\end{array}\right)$ determined by the commutation relations of $\left\{z_{n}, y_{n}\right\}$. In $A_{1}$, the Poisson brackets of each pair of elements in $Y_{T}$ are determined by the skew symmetric matrix:

$$
\left(\begin{array}{cc}
\Delta^{\prime} & U^{\prime} \\
-\left(U^{\prime}\right)^{t} & V^{\prime}
\end{array}\right)
$$

where $U^{\prime}$ is the $p \times 2$-matrix determined by the Poisson brackets between $\left\{w_{1}, \ldots, w_{p}\right\}$ and $\left\{z_{n}, y_{n}\right\},-\left(U^{\prime}\right)^{t}$ is the transpose of $U^{\prime}$ and $V^{\prime}$ is the skew symmetric matrix $\left(\begin{array}{cc}0 & \widetilde{q}_{n}^{\prime}(1) \\ -\widetilde{q}_{n}^{\prime}(1) & 0\end{array}\right)$ determined by $\left\{z_{n}, y_{n}\right\}$. Observe that each entry of $V^{\prime}$ is obtained from the corresponding entry of $V$ by derivation at $t=1$. Let us check that each $(i, j)$-entry of $U^{\prime}$ is obtained from the $(i, j)$-entry of $U$ by derivation at $t=1$. Let $U_{i}$ and $U_{i}^{\prime}$ be the $i$ th-rows of $U$ and $U^{\prime}$, respectively, 
for $1 \leq i \leq p$. If $w_{1}=z_{1}$ then $U_{1}=(1,1)$ and $U_{1}^{\prime}=(0,0)$. If $w_{1}=y_{1}$ then $U_{1}=\left(q_{1}^{-1}, \lambda_{1 n}\right)$ and $U_{1}^{\prime}=\left(-{\widetilde{q_{1}}}^{\prime}(1), \widetilde{\lambda}_{1 n}^{\prime}(1)\right)$. Hence our claim is true for $U_{1}$ and $U_{1}^{\prime}$. Suppose that, for $i=1, \ldots, p-1$, each entry of $U_{i}^{\prime}$ is obtained from the corresponding entry of $U_{i}$ by derivation at 1 . If $w_{p}=z_{k}$ for some $k<n$ then

$$
U_{p}=(1,1), \quad U_{p}^{\prime}=(0,0)
$$

If $w_{p}=y_{k}$ for some $k<n$ then

$$
U_{p}=\left(q_{k}^{-1}, \lambda_{k n}\right), \quad U_{p}^{\prime}=\left(-\widetilde{q}_{k}^{\prime}(1), \widetilde{\lambda}_{k n}^{\prime}(1)\right) .
$$

If $w_{p}=x_{k}$ for some $k<n$ then

$$
U_{p}=\left(q_{k}, \lambda_{k n}^{-1}\right), \quad U_{p}^{\prime}=\left(\widetilde{q}_{k}(1),-\widetilde{\lambda}_{k n}^{\prime}(1)\right) .
$$

Hence each $(i, j)$-entry of $U^{\prime}$ is obtained from the $(i, j)$-entry of $U$ by derivation at $t=1$.

If $T=W \cup\left\{z_{n}\right\}$ then $Y_{T}=Y_{W} \cup\left\{y_{n}\right\}=\left\{w_{1}, \ldots, w_{p}, y_{n}\right\}$. In $A_{q}$, the commutation relations of each pair of elements in $Y_{T}$ are determined by the multiplicative anti-symmetric matrix:

$$
\left(\begin{array}{cc}
\Delta & U \\
U^{*} & 1
\end{array}\right)
$$

where $U$ is the $p \times 1$-matrix determined by the commutation relations between $\left\{w_{1}, \ldots, w_{p}\right\}$ and $\left\{y_{n}\right\}$ and each $(1, \ell)$-entry of $U^{*}$ is the inverse of the $(\ell, 1)$-entry of $U$. In $A_{1}$, the Poisson brackets of each pair of elements in $Y_{T}$ are determined by the skew symmetric matrix:

$$
\left(\begin{array}{cc}
\Delta^{\prime} & U^{\prime} \\
-\left(U^{\prime}\right)^{t} & 0
\end{array}\right)
$$

where $U^{\prime}$ is the $p \times 1$-matrix determined by the Poisson brackets between $\left\{w_{1}, \ldots, w_{p}\right\}$ and $\left\{y_{n}\right\}$ and $-\left(U^{\prime}\right)^{t}$ is the transpose of $U^{\prime}$. Let $c_{\ell}$ and $d_{\ell}$ be $(\ell, 1)$-entries of $U$ and $U^{\prime}$, respectively. If $w_{\ell}=z_{k}$ for some $k<n$ then $c_{\ell}=1, d_{\ell}=0$. If $w_{\ell}=y_{k}$ for some $k<n$ then $c_{\ell}=\lambda_{k n}$, $d_{\ell}=\widetilde{\lambda}_{k n}^{\prime}(1)$. If $w_{\ell}=x_{k}$ for some $k<n$ then $c_{\ell}=\lambda_{k n}^{-1}, d_{\ell}=-\widetilde{\lambda}_{k n}^{\prime}(1)$. Hence our claim is true.

If $T=W \cup\left\{z_{n}, y_{n}\right\}$ then $Y_{T}=Y_{W} \cup\left\{x_{n}\right\}=\left\{w_{1}, \ldots, w_{p}, x_{n}\right\}$. In $A_{q}$, the commutation relations of each pair of elements in $Y_{T}$ are determined by the multiplicative anti-symmetric matrix:

$$
\left(\begin{array}{cc}
\Delta & U \\
U^{*} & 1
\end{array}\right)
$$

where $U$ is the $p \times 1$-matrix determined by the commutation relations between $\left\{w_{1}, \ldots, w_{p}\right\}$ and $\left\{x_{n}\right\}$ and each $(1, \ell)$-entry of $U^{*}$ is the inverse of the $(\ell, 1)$-entry of $U$. In $A_{1}$, the Poisson brackets of each pair of elements in $Y_{T}$ are determined by the skew symmetric matrix:

$$
\left(\begin{array}{cc}
\Delta^{\prime} & U^{\prime} \\
-\left(U^{\prime}\right)^{t} & 0
\end{array}\right)
$$

where $U^{\prime}$ is $p \times 1$-matrix determined by the Poisson brackets between $\left\{w_{1}, \ldots, w_{p}\right\}$ and $\left\{x_{n}\right\}$ and $-\left(U^{\prime}\right)^{t}$ is the transpose of $U^{\prime}$. Let $c_{\ell}$ and $d_{\ell}$ be $(\ell, 1)$-entries of $U$ and $U^{\prime}$, respectively. If $w_{\ell}=z_{k}$ for some $k<n$ then $c_{\ell}=1, d_{\ell}=0$. If $w_{\ell}=y_{k}$ for some $k<n$ then $c_{\ell}=q_{k}^{-1} \lambda_{k n}^{-1}$, $d_{\ell}=-\left(\widetilde{q}_{k}^{\prime}(1)+\widetilde{\lambda}_{k n}^{\prime}(1)\right)$. If $w_{\ell}=x_{k}$ for some $k<n$ then $c_{\ell}=q_{k} \lambda_{k n}, d_{\ell}=\widetilde{q}_{k}^{\prime}(1)+\widetilde{\lambda}_{k n}^{\prime}(1)$.

If $T=W \cup\left\{z_{n}, x_{n}\right\}$ then $Y_{T}=Y_{W} \cup\left\{y_{n}\right\}=\left\{w_{1}, \ldots, w_{p}, y_{n}\right\}$. This case was proved in the case $T=W \cup\left\{z_{n}\right\}$. 
If $T=W \cup\left\{z_{n}, y_{n}, x_{n}\right\}$ then $Y_{T}=Y_{W}$ and thus there is nothing to prove. This completes the proof.

Lemma 2.20. Retain the notation of Lemma 2.19. Let

$$
c_{\ell i}=\eta_{1}^{v_{\ell i}^{1}} \cdots \eta_{r}^{v_{\ell i}^{r}}=e_{1}(q)^{v_{\ell i}^{1}} \cdots e_{r}(q)^{v_{\ell i}^{r}}
$$

for all $1 \leq \ell, i \leq s$.

(1) The center $Z\left(\left(A_{q} /\langle T\rangle\right)\left[\mathcal{Y}_{T}^{-1}\right]\right)$ is the $\mathbb{C}$-subalgebra generated by monomials $w_{1}^{u_{1}} \cdots w_{s}^{u_{s}}$ such that $u_{1} v_{\ell 1}^{i}+\ldots+u_{s} v_{\ell s}^{i}=0$ for all $1 \leq i \leq r$. In particular, $Z\left(\left(A_{q} /\langle T\rangle\right)\left[\mathcal{Y}_{T}^{-1}\right]\right)$ is independent to $q$.

(2) The Poisson center $Z P\left(\left(A_{1} /\langle T\rangle\right)\left[\mathcal{Y}_{T}^{-1}\right]\right)$ is the $\mathbb{C}$-subalgebra generated by monomials $w_{1}^{u_{1}} \cdots w_{s}^{u_{s}}$ such that $u_{1} v_{\ell 1}^{i}+\ldots+u_{s} v_{\ell s}^{i}=0$ for all $1 \leq i \leq r$.

Proof. For $u=\left(u_{1}, \ldots, u_{s}\right) \in \mathbb{Z}^{s}$, set $w^{u}=w_{1}^{u_{1}} \cdots w_{s}^{u_{s}}$.

(1) For $f=\sum_{u} \alpha_{u} w^{u} \in\left(A_{q} /\langle T\rangle\right)\left[\mathcal{Y}_{T}^{-1}\right], f \in Z\left(\left(A_{q} /\langle T\rangle\right)\left[\mathcal{Y}_{T}^{-1}\right]\right)$ if and only if $w_{\ell} f w_{\ell}^{-1}=f$ for all $\ell$ if and only if $w^{u} \in Z\left(\left(A_{q} /\langle T\rangle\right)\left[\mathcal{Y}_{T}^{-1}\right]\right)$ for all $u$ such that $\alpha_{u} \neq 0$, and

$$
\begin{aligned}
w^{u} \in Z\left(\left(A_{q} /\langle T\rangle\right)\left[\mathcal{Y}_{T}^{-1}\right]\right) & \Leftrightarrow w_{\ell} w^{u} w_{\ell}^{-1}=w^{u} & & \text { for all } \ell=1, \ldots, s \\
& \Leftrightarrow c_{\ell 1}^{u_{1}} \cdots c_{\ell s}^{u_{s}}=1 & & \text { for all } \ell=1, \ldots, s \\
& \Leftrightarrow \prod_{i=1}^{r} \eta_{i}^{u_{1} v_{\ell 1}^{i}+\ldots+u_{s} v_{\ell s}^{i}}=1 & & \text { for all } \ell=1, \ldots, s \\
& \Leftrightarrow u_{1} v_{\ell 1}^{i}+\ldots+u_{s} v_{\ell s}^{i}=0 & & \text { for all } \ell \text { and } i .
\end{aligned}
$$

Thus $Z\left(\left(A_{q} /\langle T\rangle\right)\left[\mathcal{Y}_{T}^{-1}\right]\right)$ is the $\mathbb{C}$-algebra spanned by all monomials $w^{u}$ such that

$$
u_{1} v_{\ell 1}^{i}+\ldots+u_{s} v_{\ell s}^{i}=0
$$

for all $\ell=1, \ldots, s$ and $i=1, \ldots, r$. That is,

$$
Z\left(\left(A_{q} /\langle T\rangle\right)\left[\mathcal{Y}_{T}^{-1}\right]\right)=\left\{\sum_{u} \mathbb{C} w^{u} \mid u_{1} v_{\ell 1}^{i}+\ldots+u_{s} v_{\ell s}^{i}=0 \text { for all } 1 \leq \ell \leq s, 1 \leq i \leq r\right\} .
$$

In particular, $Z\left(\left(A_{q} /\langle T\rangle\right)\left[\mathcal{Y}_{T}^{-1}\right]\right)$ is independent to $q$.

(2) Note, by (2.2), that

$$
\widetilde{c}_{\ell i}(1)=v_{\ell i}^{1} \mu_{1}+\ldots+v_{\ell i}^{r} \mu_{r} .
$$

For $f=\sum_{u} \alpha_{u} w^{u} \in\left(A_{1} /\langle T\rangle\right)\left[\mathcal{Y}_{T}^{-1}\right], f \in Z P\left(\left(A_{1} /\langle T\rangle\right)\left[\mathcal{Y}_{T}^{-1}\right]\right)$ if and only if $\left\{w_{\ell}, f\right\} w_{\ell}^{-1}=0$ for all $\ell$ if and only if $w^{u} \in Z P\left(\left(A_{1} /\langle T\rangle\right)\left[\mathcal{Y}_{T}^{-1}\right]\right)$ for all $u$ such that $\alpha_{u} \neq 0$, and

$$
\begin{aligned}
w^{u} \in Z P\left(\left(A_{1} /\langle T\rangle\right)\left[\mathcal{Y}_{T}^{-1}\right]\right) & \Leftrightarrow\left\{w_{\ell}, w^{u}\right\}=0 & & \text { for all } \ell=1, \ldots, s \\
& \Leftrightarrow u_{1} \widetilde{c}_{\ell 1}(1)+\ldots+u_{s} \widetilde{c}_{\ell s}(1)=0 & & \text { for all } \ell=1, \ldots, s \\
& \Leftrightarrow \sum_{i=1}^{r}\left(u_{1} v_{\ell 1}^{i}+\ldots+u_{s} v_{\ell s}^{i}\right) \mu_{i}=0 & & \text { for all } \ell=1, \ldots, s \\
& \Leftrightarrow u_{1} v_{\ell 1}^{i}+\ldots+u_{s} v_{\ell s}^{i}=0 & & \text { for all } \ell \text { and } i .
\end{aligned}
$$


Hence

$$
Z P\left(\left(A_{1} /\langle T\rangle\right)\left[\mathcal{Y}_{T}^{-1}\right]\right)=\left\{\sum_{u} \mathbb{C} w^{u} \mid u_{1} v_{\ell 1}^{i}+\ldots+u_{s} v_{\ell s}^{i}=0 \text { for all } 1 \leq \ell \leq s, 1 \leq i \leq r\right\}
$$

Lemma 2.21. Retain the notation of Lemma 2.19. Let $D$ be the $\mathbb{C}$-algebra $\mathbb{C}\left[w_{1}, \ldots, w_{s}\right]$ of Lemma 2.19(1) and let $E$ be the Poisson algebra $\mathbb{C}\left[w_{1}, \ldots, w_{s}\right]$ of Lemma 2.19(2). Note that $Z\left(D\left[w_{1}^{-1}, \ldots, w_{s}^{-1}\right]\right)=Z P\left(E\left[w_{1}^{-1}, \ldots, w_{s}^{-1}\right]\right)$ by Lemma 2.20. Set

$$
Z=Z\left(D\left[w_{1}^{-1}, \ldots, w_{s}^{-1}\right]\right)=Z P\left(E\left[w_{1}^{-1}, \ldots, w_{s}^{-1}\right]\right)
$$

and $w^{u}=w_{1}^{u_{1}} \cdots w_{s}^{u_{s}}$ for $u=\left(u_{1}, \ldots, u_{s}\right) \in \mathbb{Z}^{s}$. For any $f=\sum_{u} \alpha_{u} w^{u} \in Z$ and $v \in\left(\mathbb{Z}_{+}\right)^{s}$, $w^{v} f \in D$ if and only if $w^{v} f \in E$.

Proof. In $D, w^{v} f=f w^{v}$ since $f \in Z$. Hence $w^{v} f \in D$ if and only if $v+u \in\left(\mathbb{Z}_{+}\right)^{s}$ for all $u$ such that $\alpha_{u} \neq 0$ if and only if $w^{v} f \in E$.

We recall [10, Theorem 2.4 and preceding comment]: A Poisson $\mathbb{C}$-algebra $B$ is said to satisfy the Dixmier-Moeglin equivalence if the following conditions are equivalent: For a Poisson prime ideal $P$ of $B$,

(i) $P$ is Poisson primitive.

(ii) $P$ is rational (i.e., the Poisson center of the quotient field of $B / P$ is equal to $\mathbb{C}$ ).

(iii) $P$ is locally closed (i.e., the intersection of all Poisson prime ideals properly containing $P$ is strictly larger than $P$ ).

Theorem 2.22. The multi-parameter Poisson Weyl algebra $A_{1}$ satisfies the Dixmier-Moeglin equivalence.

Proof. Since $A_{1}$ is finitely generated, it is enough to show that every rational Poisson prime ideal is locally closed by [10, Proposition 1.10 and (ii) $\Rightarrow$ (i) of the proof of Theorem 2.4]. Let $P$ be a rational Poisson prime ideal of $A_{1}$ and let $P \cap \mathcal{M}_{n}=T$. Then $T$ is an admissible set by Lemma 2.14. Since the canonical image $P^{\prime}$ of $P$ to $\left(A_{1} /\langle T\rangle\right)\left[\mathcal{Y}_{T}^{-1}\right]$ is rational, $P^{\prime}$ is locally closed by Lemma 2.19(2) and [10, Theorem 2.4]. It follows that $P$ is locally closed since admissible sets are finite.

Theorem 2.23. Let $G(Q, \Lambda)$ be a torsion free group. Then there exists a homeomorphism from Spec $R_{n}^{Q, \Lambda}$ onto P.Spec $A_{1}$ such that its restriction to $\operatorname{Prim} R_{n}^{Q, \Lambda}$ is also a homeomorphism onto P.Prim $A_{1}$.

Proof. It is enough to show the result for $A_{q}$ instead of $R_{n}^{Q, \Lambda}$ by Lemma 2.8. Let $T$ be an admissible set and set $Y_{T}=\left\{w_{1}, \ldots, w_{s}\right\}$. Denote by $\iota$ the canonical epimorphism from $A_{q}$ onto $A_{q} /\langle T\rangle$ or the canonical epimorphism from $A_{1}$ onto $A_{1} /\langle T\rangle$. For any $u=\left(u_{1}, \ldots, u_{s}\right) \in \mathbb{Z}^{s}$, we set $w^{u}=w_{1}^{u_{1}} \cdots w_{s}^{u_{s}}$ and $\iota\left(w^{u}\right)=\iota\left(w_{1}\right)^{u_{1}} \cdots \iota\left(w_{s}\right)^{u_{s}}$. We may set

$$
Z=Z\left(\left(A_{q} /\langle T\rangle\right)\left[\mathcal{Y}_{T}^{-1}\right]\right)=Z P\left(\left(A_{q} /\langle T\rangle\right)\left[\mathcal{Y}_{T}^{-1}\right]\right)
$$

by Lemma 2.20, For any prime ideal $P^{\prime}$ of $Z$, set

$$
J_{P^{\prime}}=\left\{w^{v}\left(\sum_{u \in \mathbb{Z}^{s}, \alpha_{u} \neq 0} \alpha_{u} w^{u}\right) \mid \sum \alpha_{u} \iota\left(w^{u}\right) \in P^{\prime}, v \in\left(\mathbb{Z}_{+}\right)^{s} \text { such that } v+u \in\left(\mathbb{Z}_{+}\right)^{s} \forall u\right\} .
$$


Note that $\iota\left(J_{P^{\prime}}\right)$ is the contraction of $P^{\prime}$ to $A_{q} /\langle T\rangle$ and $A_{1} /\langle T\rangle$ by Lemma 2.21 and that the ideal generated by $J_{P^{\prime}} \cup T$ is a prime ideal of $A_{q}$ and a Poisson prime ideal of $A_{1}$ by [6, 1.5] and [10, Corollary 2.3].

Let $P \in \operatorname{Spec}_{T} A_{q}$. Then there exists a unique prime ideal $P^{\prime}$ of $Z$ such that $\iota(P)$ is generated by the contraction of $P^{\prime}$ to $A_{q} /\langle T\rangle[6,1.5]$ and thus $P$ is generated by $J_{P^{\prime}} \cup T$. Similarly, if $P \in \operatorname{Spec}_{T} A_{1}$ then there exists a unique prime ideal $P^{\prime}$ of $Z$ such that $\iota(P)$ is generated by the contraction of $P^{\prime}$ to $A_{1} /\langle T\rangle$ [10, Corollary 2.3] and thus $P$ is generated by $J_{P^{\prime}} \cup T$.

Let ${ }^{\wedge}: A_{q} \longrightarrow \widehat{A}$ be the map defined by $f(q) \mapsto \widehat{f(q)}=(f(\lambda))_{\lambda \in \mathbf{K}}$. Since all $\alpha_{u}$ in (2.10) are independent to $q$ by Lemma $2.20(1), \pi_{\lambda}(\widehat{x})=x$ and $\Gamma(\widehat{x})=x$ for $x \in J_{P^{\prime}} \cup T$. Denote by $X$ the ideals of $\widehat{A}$ generated by $\widehat{J_{P^{\prime}} \cup T}$ for all $P \in \operatorname{Spec}_{T} A_{q}$ and admissible set $T$. Then the map $\widehat{\imath}: A_{q} \longrightarrow \widehat{A}$ induces a homeomorphism $\widehat{\varphi}$ from $\operatorname{Spec} A_{q}$ onto $X$ and

$$
\Gamma\left(\widehat{J_{P^{\prime}} \cup T}\right)=J_{P^{\prime}} \cup T \text {. }
$$

Hence the map $\varphi_{\Gamma}$ given in (1.4) is a homeomorphism from $X$ onto P. Spec $A_{1}$ by Corollary 1.5. It follows that $\operatorname{Spec} A_{q}$ is homeomorphic to P. Spec $A_{1}$ by the composition $\varphi_{\Gamma} \circ \widehat{\varphi}$. The restriction of this homeomorphism to the primitive spectrum Prim $A_{q}$ is also a homeomorphism onto the Poisson primitive spectrum P.Prim $A_{1}$ by [5, 9.4], [7, 5.6] and Theorem 2.22.

Let $\mathcal{O}(V)$ be the coordinate ring of a Poisson affine variety $V$. The sets $\left\{p \in V \mid \mathcal{P}\left(\mathfrak{m}_{p}\right)=P\right\}$ for $P \in \mathrm{P}$. Prim $\mathcal{O}(V)$ are called the symplectic cores in $V$, where $\mathfrak{m}_{p}=\{f \in \mathcal{O}(V) \mid f(p)=0\}$. Refer to [5, §6] for symplectic cores.

Corollary 2.24. Let $G(Q, \Lambda)$ be a torsion free group. Then $\operatorname{Prim} R_{n}^{Q, \Lambda}$ is homeomorphic to the space of symplectic cores in the affine variety $\mathbb{C}^{n}$, where the Poisson bracket in $\mathbb{C}^{n}$ is given by $A_{1}$.

Proof. It follows immediately by Theorem 2.22, Theorem 2.23 and [5, Lemma 9.3].

\section{REFERENCES}

1. M. Akhavizadegan and David A. Jordan, Prime ideals of quantized Weyl algebras, Glasgow Math. J. 38 (1996), 283-297.

2. K. A. Brown and K. R. Goodearl, Lectures on algebraic quantum groups, Advanced courses in mathematicsCRM Barcelona, Birkhäuser Verlag, Basel-Boston·Berlin, 2002.

3. J. Dixmier, Enveloping algebras, The 1996 printing of the 1977 English translation Graduate Studies in Mathematics, vol. 11, American Mathematical Society, Providence, 1996.

4. K. R. Goodearl, A Dixmier-Moeglin equivalence for Poisson algebras with torus actions, in Algebra and Its Applications (D. V. Huynh, S. K. Jain, and S. R. Lopez-Permouth, Eds.) Contemp. Math. 419 (2006), $131-154$.

5. _ Semiclassical limits of quantized coordinate rings, in Advances in Ring Theory (D. V. Huynh and S. R. Lopez-Permouth, Eds.) Basel Birkhäuser (2009), 165-204.

6. K. R. Goodearl and E. S. Letzter, Prime and primitive spectra of multiparameter quantum affine spaces, in Trends in Ring Theory Proc. Miskolc. Conf. 1996 (V. Dlab and L. Márki, eds.), Canad. Math. Soc. Conf. Proc. Series (1998), 39-58.

7. Amer. Math. Soc. 352 (2000), 1381-1403.

8. David A. Jordan and Sei-Qwon Oh, Poisson brackets and Poisson spectra in polynomial algebras, New Trends in Noncommutative Algebra, Contemp. Math. 562 (2012), 169-187.

9. G. Maltsiniotis, Groupes quantique et structures différentielles, C.R. Acad. Sci.Paris Ser. I Mat. 311 (1990), 831-834. 
10. Sei-Qwon Oh, Symplectic ideals of Poisson algebras and the Poisson structure associated to quantum matrices, Comm. Algebra 27 (1999), 2163-2180.

11. _ Quantum and Poisson structures of multi-parameter symplectic and Euclidean spaces, J. Algebra 319 (2008), 4485-4535.

12. Sei-Qwon Oh, Chun-Gil Park, and Yong-Yeon Shin, Quantum n-space and Poisson n-space, Comm. Algebra 30(9) (2002), 4197-4209.

13. Sei-Qwon Oh and Mi-Yeon Park, Relationship between quantum and Poisson structures of odd dimensional Euclidean spaces, Comm. Algebra 38(9) (2010), 3333-3346.

Department of Mathematics, Chungnam National University, 99 Daehak-ro, Yuseong-Gu, DaeJEON 34134, KOREA

E-mail address: sqoh@cnu.ac.kr 